\title{
Restricting the Vertical and Horizontal Extent of the Field-of-View: Effects on Manoeuvring Performance
}

\author{
Sander Edward Michiel Jansen ${ }^{*}, 1,2$, Alexander Toet ${ }^{2}$ and Nicolaas Johannes Delleman ${ }^{3}$ \\ ${ }^{I}$ Center for Advanced Gaming and Simulation, Utrecht University, The Netherlands \\ ${ }^{2}$ TNO Human Factors, Soesterberg, The Netherlands \\ ${ }^{3}$ Faculty of Movement Science, VU University, Amsterdam, The Netherlands
}

\begin{abstract}
It is known that Field-of-view restrictions affect distance estimation, postural equilibrium, and the ability to control heading. These are all important factors when manoeuvring on foot through complex structured environments. Although considerable research has been devoted to the horizontal angular extent of the Field-of-View (FoV), rather less attention has been paid to the vertical angle. The present study investigated the effects of both vertical and horizontal FoV restriction on manoeuvring performance and head movement while traversing an obstacle course consisting of three different types of obstacles. A restriction of both the horizontal and vertical angle of the visual field resulted in increased time needed to traverse the course. In addition, the extent of head movement during traversal was affected by vertical, but not horizontal viewing restriction. Furthermore, it was investigated if performance could be improved by altering the orientation of the visual field instead of its dimensions. The results do not indicate this. The findings of this study can be used to formulate requirements for the selection and development of field-of-view limiting devices, such as head-mounted displays and night-vision goggles.
\end{abstract}

Keywords: Field-of-view, obstacle avoidance, locomotion, head-mounted displays.

\section{INTRODUCTION}

Head-mounted displays (HMDs) are frequently used for training and rehearsing tasks in virtual environments which involve human locomotion through complex structured environments (e.g. dismounted soldiers, first responders). A central issue in the design and selection of HMDs is the field-of-view (FoV). Unrestricted, the human $\mathrm{FoV}$ has an average horizontal angle of $200^{\circ}$ and an average vertical angle of $135^{\circ}$ [1]. However, most commercially available HMDs present the user with a $40-70^{\circ}$ horizontal angle, combined with a vertical angle that often does not exceed $50^{\circ}$. Large displays are more complex and costly. Therefore, it is useful to understand the effects of FoV restriction on a given task in order to produce or select the display that optimises performance and costs.

It has been shown that under full cue conditions, people can accurately judge the distances of targets resting on the ground up to 25 meters [2]. However, when the FoV is restricted, this causes underestimation of target distance, both in real $[3,4]$ and virtual environments $[5,6]$.

Next to impaired distance estimation, the loss of input from the peripheral visual field also causes a decrease in the maintenance of postural equilibrium [7] and the ability to control heading [8]. When manoeuvring through complex structured environments, all of these tasks (estimating distance, maintaining balance, controlling heading) are

\footnotetext{
*Address correspondence to this author at the Center for Advanced Gaming and Simulation, Utrecht University, The Netherlands; Tel: +31 6222296 65, +31 3025354 90; Fax: +31 3025346 19; E-mail: sander.jansen@tno.nl
}

important. Therefore, it is useful to understand how manoeuvring behaviour through such environments is affected by FoV-restriction.

Previous work by the authors $[9,10]$ showed that both speed and accuracy of moving through a complex environment increased as the horizontal angle of the visual field was enlarged to $75^{\circ}$. Surprisingly, further enlargement (to $120^{\circ}$ ) did not yield any performance improvement. This interesting finding gave rise to the idea that the restricted vertical angle (which was set at $50^{\circ}$ ) might play an important role in the impairment of performance for such manoeuvring tasks.

Although considerable research has been devoted to the horizontal angular extent of the visual field, rather less attention has been paid to the vertical angle. However, a few studies explored the effects of loss of sight of one's lower limbs on task performance. $\mathrm{Wu}$ and colleagues [11] for instance, observed impaired performance on a distance estimation task when restricted vertically, and found restored values when participants were allowed to make head movements. In addition, Rietdyk and Rhea [12] studied the effects of exproprioceptive (sight of own limbs) and exteroceptive (cues in the environment) information on obstacle crossing. They conclude that information about obstacle position and size is used in advance to plan a manoeuvre, while information about the body relative to the obstacle is used to control and update movement during the execution [13]. Patla and Vickers [14] identified two dominant gaze behaviours during adaptive locomotion: landing target fixation and travel gaze fixation. They argue that the latter is more dominant and consists of the eyes being directed at the ground ahead and not to a specific 
location. Furthermore, Marigold and Patla [15] reported increased head pitch angle as a result of a blocked lower visual field. Taken together, these findings show the importance of the lower visual field in adaptive locomotion.

From an applied perspective, it would be of interest to know the extent of the performance degrading effects caused by FoV-restricting devices such as HMDs and night-vision goggles. By systematically investigating how both the horizontal and vertical angle influence complex behaviour, it will be possible to determine requirements for the selection and development of these FoV limiting devices. It would be useful to know how much of the performance degradation caused by horizontal FoV-restriction can be compensated for by an increase of the vertical angle and vice versa. A recent preliminary study showed that enlarging the vertical angle from $18^{\circ}$ to $48^{\circ}$ yields a greater performance increase on a complex locomotion task than enlarging the horizontal angle from $75^{\circ}$ to $180^{\circ}[16]$.

The present study aims to investigate this relationship systematically by fully combining five vertical with four horizontal angles resulting in 20 combinations ranging from a very small to a fully unrestricted FoV. We will explore three different questions. First, we examine the effects of both horizontal and vertical FoV-restriction on manoeuvring performance within a complex structured course. To complete this course, three different types of obstacles need to be overcome. Each of these will require different bodily movements in order to cross them. Performance will be measured as the time needed to traverse the course as well as the number of errors made. It is expected that both horizontal and vertical FoV-restriction will decrease human performance during a manoeuvring task and that this will become manifest as an increase of both the time to complete the course and the number of errors made.

Second, a head-tracker will be used to investigate the influence of FoV-restriction on head movement during locomotion. It is expected that participants will increase the number and extent of head movements to compensate for loss of peripheral vision. Specifically, we expect increased pitch rotation for vertical field-of-view restrictions and increased yaw rotation for horizontal restrictions.

Third, we will investigate if the orientation of the visual field has an effect on performance. We therefore examine the effects of an upward, centred and downward oriented view on task performance. Such potential performance differences may suggest an alternative way to optimise performance with visual field restricting devices.

\section{MATERIALS AND METHODOLOGY}

\subsection{Participants}

The procedures of this study were approved by the TNO Human Factors internal review board on experiments with human participants. Seventeen paid participants $(8$ male and 9 female) with an average age of $23.6(S D=8.7)$ gave informed consent to take part in the experiment. All were free of any known neurological or orthopaedic disorders, or any impediments to normal locomotion, as verified by selfreport. Furthermore, all participants had normal (20/20) or corrected-to-normal vision.

\subsection{Apparatus}

\subsubsection{Goggles}

For each combination of a horizontal $\left(40^{\circ}, 80^{\circ}, 115^{\circ}\right.$ and $\left.200^{\circ}\right)$ and a vertical $\left(25^{\circ}, 40^{\circ}, 60^{\circ}, 90^{\circ}\right.$ and $\left.135^{\circ}\right)$ angle, a separate pair of safety goggles was used (type Bollé Targa; www.bolle-safety.com). To restrict the view to a certain extent of the visual field, part of the lens was covered with duct tape (see Fig. (1) for a number of examples). Also, four extra pairs of goggles (two upward and two downward oriented) were prepared to investigate the effects of visual field orientation on task performance. This was done for the $80^{\circ} \times 90^{\circ}$ and $115^{\circ} \times 60^{\circ}$ conditions $(\mathrm{H} \mathrm{x} \mathrm{V})$. A total of 24 pairs of goggles were used in this study $(4 \times 5$ viewing angles and $2 \times 2$ orientations).

\subsubsection{Environment}

The obstacle course was a straight pathway (length 850 $\mathrm{cm}$, width $140 \mathrm{~cm}$ ), flanked by wooden frames covered with light-coloured linen sheets. Three obstacles were evenly spaced over the length of the course. Each of them required the performance of different bodily movements in order to cross them. The first obstacle consisted of three thin wooden boards, with heights of 20,30 and $40 \mathrm{~cm}$, placed upright on the ground across the entire width of the course. The second obstacle consisted of three room-dividing walls, placed parallel to each other. To traverse this segment of the course, participants had to follow an S-curved trajectory through a

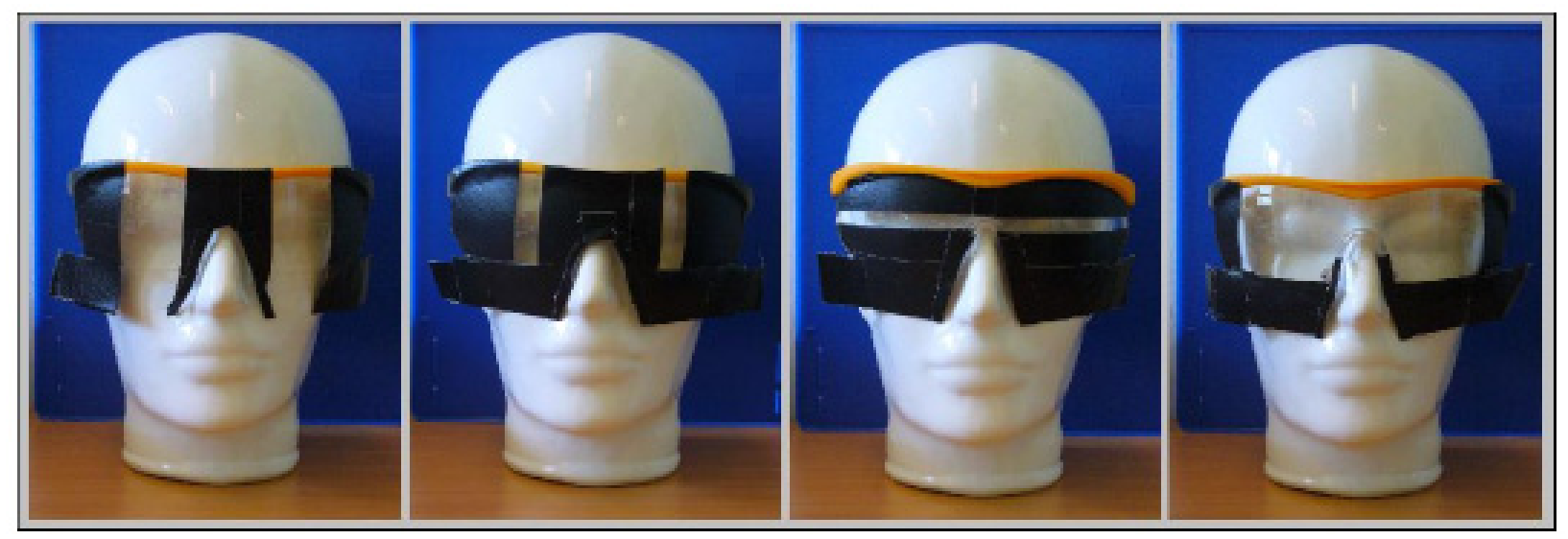

Fig. (1). Four examples of FoV limiting goggles. 
$60 \mathrm{~cm}$ wide passage between each two walls. The third obstacle consisted of a low hanging bar (at $110 \mathrm{~cm}$ above the ground) extending across the entire width of the course. Participants needed to duck to get underneath it. The bar was made from soft material (polyethylene foam) to prevent injury in case of collision. A future study is planned, in which the present experiment will be replicated in a virtual environment. Therefore, the visual structure of the obstacle course was intentionally kept simple and the view of the outside world was blocked by enclosing the course. These measures make it easier for the experimental environment to be modelled in a virtual world. See Fig. (2) for a schematic representation with photos.

\subsubsection{Time Registration}

To register the time that participants needed to traverse the course, four pairs of poles equipped with infrared lightemitting diodes and photoelectric beam sensors were used (type Velleman PEM5D; www.velleman.de). The grey squares in Fig. (2) indicate their position within the course. One pole emits and registers the return of an infrared light beam, which is reflected by a mirror on its companion (opposite) pole. The moment of interruption of the beam by a participant was registered. From this, the time needed to traverse the entire course could be computed.

\subsubsection{Video Registration}

Four surveillance cameras recorded all trials. Three of these registered different parts of the course, while the fourth filmed an overview of the entire track by using a fish-eye lens. The videos were used to count the number of errors made by participants as well as to observe certain qualitative aspects of the manoeuvring behaviour.

\subsubsection{Head-Tracking}

To register the head movements made by participants during the traversal of the course, a movement registration system was used that registered the orthogonal linear acceleration and the angular velocity of the roll, pitch and yaw rotation of the head. The system consists of a sensory device housing six Murata Gyrostar sensors, which was connected to a data logger using a sampling rate of $50 \mathrm{~Hz}$. Every trial was registered as a separate data file. The sensory device was attached to a headband worn by the participants. The data logger was placed in a bag worn around the waist. The intermediate time registrations were used to investigate the extent of head rotation for each of the segments

\subsection{Design and Procedures}

A 4 (horizontal angle) x 5 (vertical angle) withinparticipants design was used, with an unrestricted (without goggles) condition both at the beginning and end of the experiment. Both variables were randomized across trials using a Latin square design [17], since these were assumed to influence the data collection. In addition, the four extra conditions (orientation) were distributed randomly within each participant's trial-set.

Each participant traversed the course with a total of 26 different viewing conditions (i.e. 4 horizontal angles $\mathrm{x} 5$ vertical ones, 2 unrestricted and 4 orientation conditions). After filling out the informed consent form, participants were instructed to traverse the course with each of the conditions. For each trial, a number of performance measurements were registered:

1. The time that was needed to traverse the entire course.

2. The angular extent of head movement for all three axes (pitch, roll and heading).

3. The number of errors for each trial.

Participants were told that it was important not to touch any of the objects constituting the course, thus simulating a potentially dangerous environment. This instruction served to keep the error count at a low level. A small break was held after half of the trials had been recorded, during which

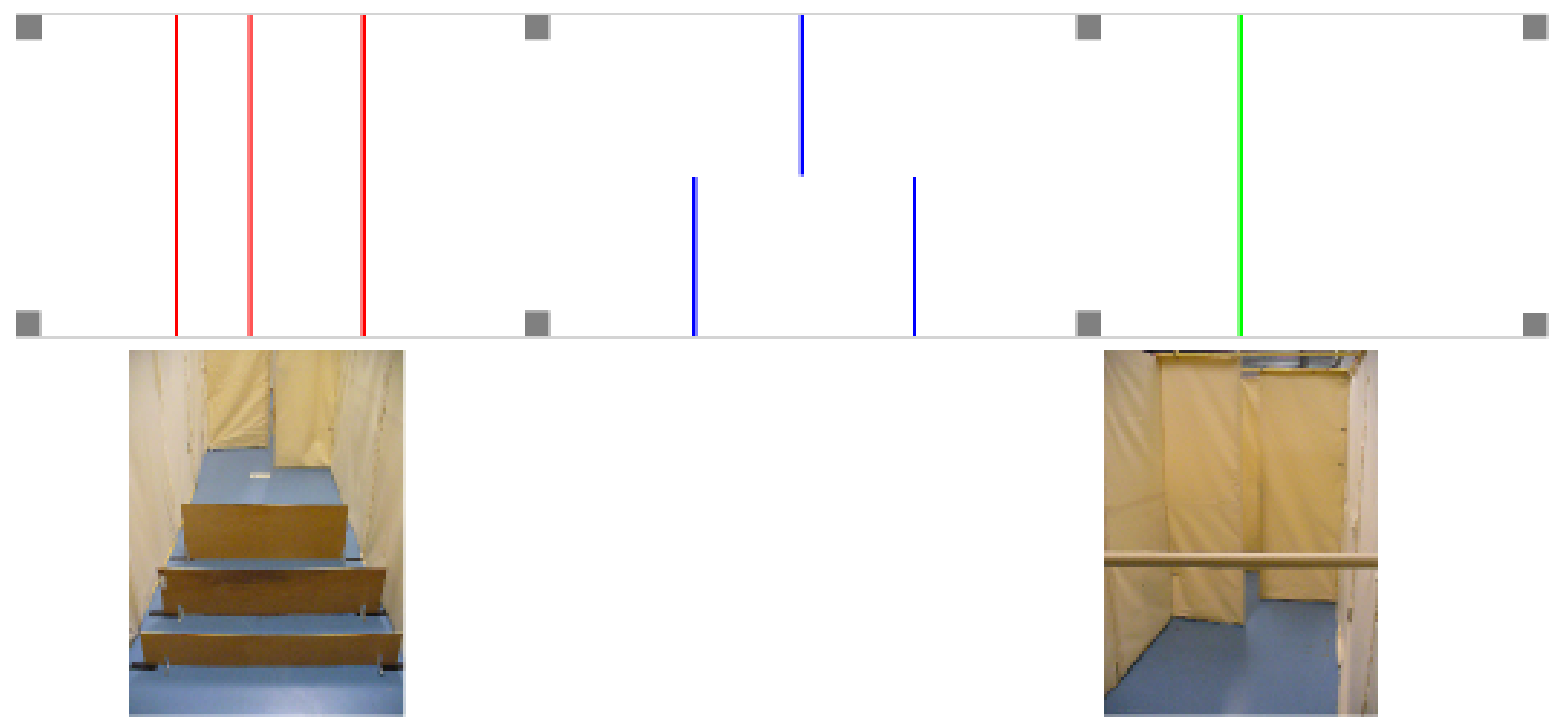

Fig. (2). A top view schematic representation of the experimental setup. From left to right, lines represent thin wooden boards, walls and lowing hanging bar, respectively. Furthermore, grey squares represent the time measurement poles. Left photgraph: The three thin wooden boards obstacle. Right photograph: The room-dividing walls obstacle in the background and the suspended bar obstacle in the foreground. 
participants could drink something and go to the bathroom.

\subsection{Statistical Analyses}

Mauchley's sphericity test was performed for each ANOVA. Whenever this revealed a violation of the sphericity assumption, The Greenhouse-Geisser correction was applied. Also in such a case, Bonferroni's post hoc procedure was used instead of Fisher's LSD to compare pairwise means [18]. All analyses were performed with STATISTICA 8.0 and significance levels were set to $5 \%$.

\section{RESULTS}

\subsection{FoV}

\subsubsection{Effects of FoV on Time}

Because of the high degree of variability in speed between the participants, the time measurement used in this experiment was defined as the percentual increase compared to each participant's unrestricted condition (when not wearing goggles). Fig. (3) shows the time increase as a function of horizontal and vertical viewing angle. The horizontal angular extent had a main effect on time, $F(3,36)$ $=8.532, p<.01$ with pair wise differences showing significance only between the smallest angle (i.e. $40^{\circ}$ ) and each of the other angles. Furthermore, the extent of the vertical angle had a main effect on time as well, $F(4,48)=$ 9.941, $p<.01$. Significant pair wise differences exist between the $25^{\circ}$ condition and each of the other angles.

\subsubsection{Effects of FoV on Error Count}

Error count was not affected by FoV. However, there was a significant difference in error count between the three tasks: $57 \%$ of all errors were made during the stepping over task, compared to $23 \%$ during the avoiding walls segment and $20 \%$ during ducking.

\subsubsection{Effects of FoV on Head Movement}

Decreasing the horizontal viewing angle had no significant effect on total yaw rotation, $F(3,33)=.978, p<$ .35 . On the other hand, decreasing the vertical angle did cause an increase in pitch rotation, $F(4,44)=10.763, p<$ .001 . The interaction between vertical angle and task was also significant, $F(8,88)=3.727, p<.001$. Pitch rotation

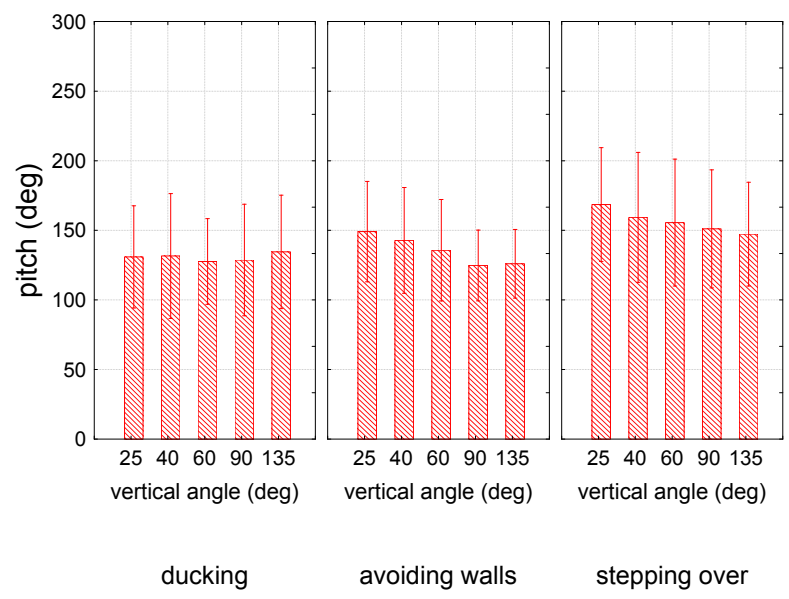

during the avoiding walls and stepping over tasks were influenced by the limitition of the angle, while the pitch rotation during the ducking task was not (see Fig. 4).

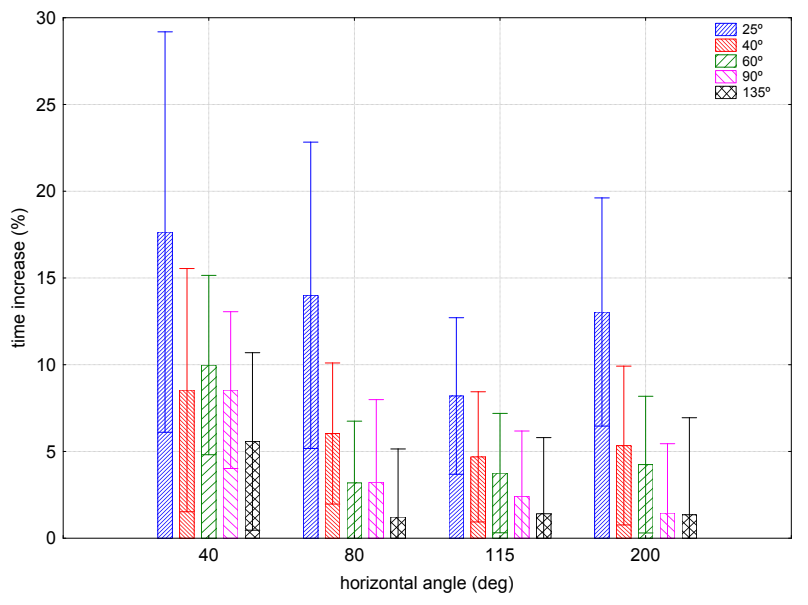

Fig. (3). Percentual increase of time needed to traverse the entire course as a function of horizontal and vertical viewing angle. Colored bars indicate vertical angles and error bars denote 0.95 confidence intervals.

\subsection{Orientation}

\subsubsection{Effects of Orientation on Time}

Time to complete the course was affected by viewing orientation, $F(2,30)=5.164, p=.012$ (see Fig. 5). Pairwise comparison revealed a difference between an upward oriented and centered view $(p<.01)$, as well as between a upward and downward oriented view $(p<.01)$. Furthermore, it seems that a downward view increases overall time compared to a centered view. However this did not reach statistical significance $(p=.28)$.

\subsubsection{Effects of Orientation on Error Count}

A significant interaction exists between viewing orientation and task, $F(4,60)=3.494, p=.012$. Performing the stepping over task with an upward oriented view caused more errors than with a centered view $(p=0.015)$. This was not the case with the other tasks.

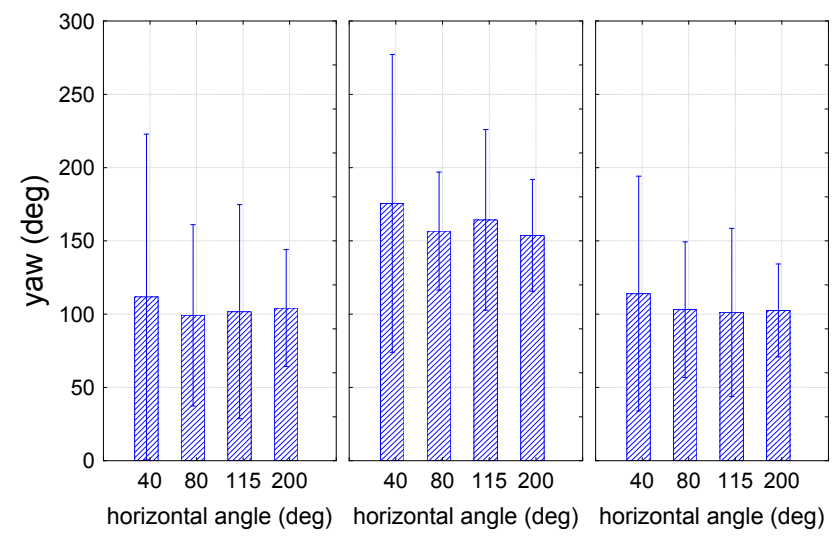

ducking avoiding walls stepping over

Fig. (4). Left: Total pitch rotation of the head as a function of vertical angular extent and task. Right: Total yaw rotation of the head as a function of horizontal angular extent and task. Error bars denote 0.95 confidence intervals. 


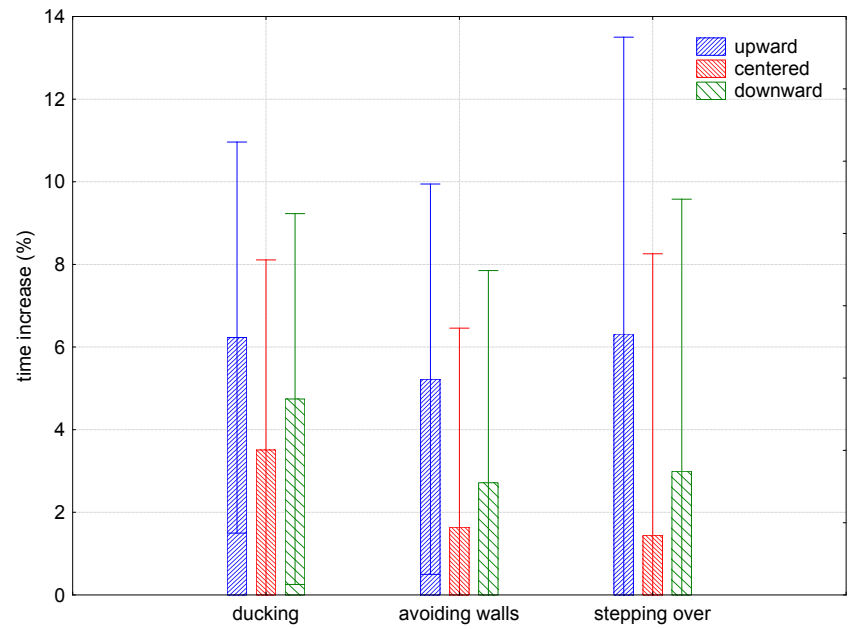

Fig. (5). Mean time increase (compared to unrestricted condition) as a function of viewing orientation and task. Error bars denote 0.95 confidence intervals.

\subsubsection{Effects of Orientation on Head Movement}

Viewing orientation had no effect on yaw $(F(2,14)=$ $2.530, p=.012)$ and pitch $(F(2,14)=.241, p=.79)$ rotation of the head.

\section{DISCUSSION}

The present study investigated a number of research questions, which will be discussed here independently. First we hypothesised that FoV-restriction would cause performance degrading effects on manoeuvring tasks through a structured environment. According to Rieser and colleagues [19], a large visual field is required to create and maintain an accurate representation of the world. Limitation of this field by reducing either the horizontal or vertical viewing angle produces an impairment of this representation, which results in impaired perceptuomotor performance. We expected that a reduction of either the horizontal or the vertical viewing angle would become manifest as an increase in elapsed time and error count in our experimental paradigm. Indeed, the results indicate that both a limitation of the horizontal and the vertical viewing angle caused participants to move slower through the course. However, it can not be concluded from this study that this causes increased error rate.

We first looked at the main effect of the horizontal viewing angle on elapsed time. A reduction of the horizontal extent of the visual field yields an increase in time needed to complete the course. An interesting finding was the unanticipated high elapsed time of the specific FoV of $200^{\circ}$ $x 25^{\circ}(\mathrm{HxV})$. A possible explanation for this finding could be that the ratio between the horizontal and vertical angle is very unnatural (a very narrow slit), which results in slower movement. Apart from this specific condition, the overall findings are consistent with previous studies, which showed impaired performance caused by horizontal FoV-restriction on manoeuvring tasks $[9,10]$, distance estimation $[3,5]$ and postural equilibrium [7]. In these cases the vertical angle was kept constant, usually at an extent often seen in current FoVrestricting devices (i.e. $30-50^{\circ}$ ).
The present study also investigated the effects of reduced vertical angular extent. The results show that decreasing the vertical angle results in increased time needed to complete the course. Specifically, performance with a small vertical angle $\left(25^{\circ}\right)$ differs from that with larger ones. These results were similar for all three tasks (i.e. stepping over, avoiding walls, and ducking), suggesting that the effects are robust and do not depend on the nature of the actual bodily movements needed for each manoeuvring task. In addition, it is interesting to look at the interaction between horizontal and vertical angle restriction. For instance, enlarging a FoV of $80^{\circ} \times 40^{\circ}(\mathrm{HxV})$ can be done by increasing either the horizontal or vertical extent of the visual field. When enlarging to $115^{\circ} \mathrm{x} 40^{\circ}$ by increasing the horizontal angle, performance improves by $1.34 \%$. On the other hand, when enlarging the vertical component to create a FoV of $80^{\circ} \mathrm{x}$ $60^{\circ}$, the performance improves by $2.84 \%$. This confirms previous findings, which showed that an enlargement of the vertical angle from $18^{\circ}$ to $48^{\circ}$ results in greater performance improvement than an enlargement of the horizontal angle from $75^{\circ}$ to $180^{\circ}[16]$. The results presented here should encourage further investigation of the effects of vertical FoV-restriction.

The second hypothesis concerned the influence of FoV restriction on head movements. It was expected that participants would compensate for the loss of peripheral visual information by making extensive head movements. More specifically, it was expected that a decrease of the horizontal angle would cause an increase of the extent of yaw rotation of the head, while reduction of the vertical angle would result in increased pitch rotation. The results show that a horizontal restriction does not have an effect on head movement, but a vertical restriction does. During the avoiding walls and stepping over tasks especially, pitch rotation is increased with vertical restriction. These tasks required participants to estimate distances between obstacles, which is facilitated by integrating ground surfaces [11]. Therefore, a decrease of the vertical angular extent requires increased pitch rotation of the head. Ducking is least influenced by FoV restriction. A possible explanation for this could be that the manoeuvre needed to avoid this obstacle did not require a view of the obstacle during the actual ducking. Instead, it seems that participants planned this manoeuvre beforehand and could then perform the actual movement without visual feedback. The other two tasks (stepping over and avoiding walls) demanded visual information during the execution to control heading and foot placement in order to avoid collision. Therefore, visual field restriction did cause increased head movement for these tasks. Information about the extent and speed of head movement is useful in determining the type of tracker that is needed in order to minimize lag in HMDs, which is an important issue when interacting within a virtual environment. According to Keller and Colucci [20], most HMDs used nowadays have a $60-90 \mathrm{~ms}$ lag, whereas $16 \mathrm{~ms}$ is already perceivable.

Besides the effects of FoV restriction, we explored the effect of visual field orientation on task performance and head movement. From the results it can be concluded that an upward oriented view yields impaired performance as 
compared to a centred view. This became manifest as an increase in elapsed time. Also, during the stepping over task, more errors were made with an upward oriented view. This is in line with work by Mon-Williams and colleagues [21], who state that high gaze angles quickly cause visual fatigue, which may account for the performance decrements found here. Another cause could be the disturbance of the vestibular system that is caused by the altered head orientation, which is needed to compensate for the change in visual field orientation. Furthermore, it seems that a centred view has a slight advantage over a downward oriented view but this could not be confirmed by a statistical analysis due to the small amount of data that was gathered to investigate this question. The pilot was done to explore the possibility of display orientation instead of display enlargement as a means to improve task performance in virtual environments. At this point, the results do not suggest that an alternative orientation would increase performance during a manoeuvring task, but a more elaborate investigation is preferable.

In summary, the findings of the present study can be used to formulate requirements for the selection and development of field-of-view limiting devices, such as head-mounted displays and night-vision goggles, which use head-tracking sensors to update the images presented to the user. It should be noted that the findings of this study are restricted to a controlled laboratory setting which involves simple manoeuvring tasks. It would be of interest to see if these results could be replicated in a larger, more complex environment in which additional tasks need to be performed. Also, other performance measurements need to be considered which could be used to analyse the qualitative aspects of manoeuvring performance. A possible method could be full-body motion capture.

\section{ACKNOWLEDGMENTS}

This research has been supported by the gate project, funded by the Netherlands Organization for Scientific Research (nwo) and the Netherlands ict Research and Innovation Authority (ict Regie).

\section{REFERENCES}

[1] Werner EB. Manual of Visual Fields. New York: Churchill Livingstone 1991.

[2] Loomis JM, Fujita N, Da Silva JA, Fukusima SS. Visual space perception and visually directed action. J Exp Psychol Hum Percept Perform 1992; 1(4): 921.
[3] Watt SJ, Bradshaw MF, Rushton SK. Field of view affects reaching, not grasping. Exp Brain Res 2000; 135(3): 411-6.

[4] Willemsen P, Colton MB, Creem-Regehr SH, Thompson WB. The effects of head-mounted display mechanical properties and Veld of view on distance judgments in virtual environments. ACM Trans Appl Percept 2009; 6(2): 1-14.

[5] Fortenbaugh FC, Hicks JC, Hao L, Turano KA. Losing sight of the bigger picture: Peripheral field loss compresses representations of space. Vision Res 2007; 47(19): 2506-20.

[6] Arthur KW. Effects of field of view on performance with headmounted displays dissertation. University of North Carolina 2000.

[7] Turano K, Herdman S, Dagnelie G. Visual stabilization of posture in retinitis pigmentosa and in artiVcially restricted visual fields. Invest Ophthalmol Vis Sci 1993; 34(10): 3004-10.

[8] Patterson R, Winterbottom MD, Pierce BJ. Perceptual issues in the use of head-mounted visual displays human factors. J Hum Factors Ergon Soc 2006; 48(3): 555-73.

[9] Toet A, Jansen S, Delleman N. Effects of field-of-view restrictions on speed and accuracy of manoeuvring. Percept Mot Skills. 2007; 105(3 Pt 2): 1245-56.

[10] Toet A, Jansen S, Delleman N. Effects of field-of-view restrictions on manoeuvring in a 3-D environment. Ergonomics 2008; 51(3): 385-94.

[11] Wu B, Ooi T, He Z. Perceiving distance accurately by a directional process of integrating ground information. Nature 2004; 428(6978): 73-7.

[12] Rietdyk S, Rhea CK. Control of adaptive locomotion: effect of visual obstruction and visual cues in the environment. Exp Brain Res 2006; 169(2): 272-8.

[13] Rhea CK, Rietdyk S. Visual exteroceptive information provided during obstacle crossing did not modify the lower limb trajectory. Neurosci Lett 2007; 418(1): 60-5.

[14] Patla AE, Vickers JN. How far ahead do we look when required to step on specific locations in the travel path during locomotion? Exp Brain Res 2003; 148(1): 133-8.

[15] Marigold DS, Patla AE. Visual information from the lower visual field is important for walking across multi surface terrain. Exp Brain Res 2008; 188(1): 23-31.

[16] Toet A, Van Der Hoeven M, Kahrimanović M, Delleman N. Effects of field-of-view on human locomotion. Proc SPIE 2008; 6955: $69550 \mathrm{H}$

[17] Wagenaar WA. Note on the construction of digram-balanced Latin squares. Psychol Bull 1969; 72(6): 384-386.

[18] Field A. Discovering statistics using SPSS. London, UK: Sage Publications Ltd 2009.

[19] Rieser J, Hill E, Talor C, Bradfield A, Rosen S. Visual experience visual, field size and the development of nonvisual sensitivity to the spatial structure of outdoor neighborhoods explored by walking. J Exp Psychol Gen 1992; 121(2): 210-21.

[20] Keller K. Perception in HMDs: what is it in head-mounted displays (HMDs) that really makes them all so terrible? Proc SPIE 1998; 3362: 46.

[21] Mon-Williams M, Burgess-Limerick R, Plooy A, Wann J. Vertical gaze direction and postural adjustment: an extension of the Heuer model. J Exp Psychol Appl 1999; 5: 35-53. 